\title{
Anticorrelated Hard/Soft X-Ray Emission from the X-Ray Burster 4U 0614+091
}

\section{Citation}

Ford, E., P. Kaaret, M. Tavani, B. A. Harmon, S. N. Zhang, D. Barret, J. Grindlay, P. Bloser, and R. A. Remillard. 1996. "Anticorrelated Hard/Soft X-Ray Emission from the X-Ray Burster $4 \mathrm{U}$ 0614+091." The Astrophysical Journal 469 (1): L37-40. https://doi.org/10.1086/310265.

\section{Permanent link}

http://nrs.harvard.edu/urn-3:HUL.InstRepos:41399840

\section{Terms of Use}

This article was downloaded from Harvard University's DASH repository, and is made available under the terms and conditions applicable to Other Posted Material, as set forth at http:// nrs.harvard.edu/urn-3:HUL.InstRepos:dash.current.terms-of-use\#LAA

\section{Share Your Story}

The Harvard community has made this article openly available.

Please share how this access benefits you. Submit a story.

Accessibility 
The Astrophysical Journal, 469:L37-L40, 1996 September 20

(c) 1996. The American Astronomical Society. All rights reserved. Printed in U.S.A.

\title{
ANTICORRELATED HARD/SOFT X-RAY EMISSION FROM THE X-RAY BURSTER 4U 0614+091
}

\author{
E. Ford, P. KAARET, AND M. TAVANI \\ Department of Physics and Columbia Astrophysics Lab, Columbia University, 538 West 120th Street, New York, NY 10027; eric@orphee.phys.columbia.edu \\ B. A. HARMON \\ NASA/Marshall Space Flight Center, ES 84, Huntsville, AL 35812 \\ S. N. ZHANG \\ University Space Research Association/Marshall Space Flight Center, ES 84, Huntsville, AL 35812 \\ D. Barret, J. Grindlay, AND P. Bloser \\ Harvard Smithsonian Center for Astrophysics, 60 Garden Street, Cambridge, MA 02138 \\ AND \\ R. A. Remillard \\ Center for Space Science, Massachusetts Institute of Technology, Room 37-595, Cambridge, MA 02139; rr@space.mit.edu \\ Received 1996 June 25; accepted 1996 July 16
}

\begin{abstract}
We have detected transient X-ray activity from the X-ray burster 4U 0614+091 simultaneously with BATSE on board the Compton Gamma Ray Observatory (20-100 keV) and the all-sky monitor (ASM) on board the Rossi $X$-Ray Timing Explorer (1-12 keV). The peak fluxes reach approximately $40 \mathrm{mcrab}$ in both instruments over a period of about 20 days. The variable emission shows a clear anticorrelation of the hard X-ray flux with the soft $\mathrm{X}$-ray count rate. The observed anticorrelation is another clear counterexample to the notion that only black hole binaries exhibit such correlations. The individual spectra during this period can be fitted by power laws with photon indices $2.2 \pm 0.3(\mathrm{ASM})$ and $2.7 \pm 0.4$ (BATSE), while the combined spectra can be described by a single power law with index $2.09 \pm 0.08$. BATSE and the Rossi X-Ray Timing Explorer ASM are a good combination for monitoring X-ray sources over a wide energy band.

Subject headings: accretion, accretion disks — stars: individual (4U 0614+091) — stars: neutron —

X-Rays: Stars
\end{abstract}

\section{INTRODUCTION}

Many X-ray emission properties once thought to uniquely characterize black hole candidates have now been observed in systems containing weakly magnetized neutron stars (e.g., Barret \& Vedrenne 1994; van der Klis 1994; Inoue 1990). A classic observational feature of black hole candidates (BHCs) has been the presence of hard power-law tails in the energy spectra extending above $100 \mathrm{keV}$. Typically, the hard X-ray emission is anticorrelated with soft X-ray luminosity (Tanaka 1989). Measurements below $20 \mathrm{keV}$ show that X-ray bursters, representing weakly magnetized neutron stars (NSs), also have power-law tails. As in the BHCs, an anticorrelation of flux and hardness is clearly observed in a number of bursters: e.g., 4U 1636-536 (Breedon et al. 1986), 4U 1735-444 (Smale et al. 1986), 4U 1705-44 (Langmeier et al. 1987), 4U 1608-522 (Mitsuda et al. 1989), and 4U 0614+091 (Barret \& Grindlay 1995).

Until recently, most observations of accreting NSs have been limited to less than $20 \mathrm{keV}$. A notable exception was the detection of a 150 day outburst from the burster Cen X-4 with SIGNE 2 MP/PROGNOZ 7 (Bouchacourt et al. 1984) in the 3-163 keV band. In this observation, the hard X-ray flux was high, both at the beginning and end of the outburst, when the soft X-ray flux was low. With observations by SIGMA, the situation has changed and many more X-ray bursters are now detected above $20 \mathrm{keV}$ (Barret \& Vedrenne 1994). The hard $\mathrm{X}$-ray emission is typically variable on the timescale of days and seems to be detected preferentially from the low-luminos- ity bursters. Additional bursters are being detected in hard X-rays with BATSE monitoring (see Barret et al. 1996). Numerous X-ray bursters are now firmly established as sources of hard X-ray emission.

$4 \mathrm{U} 0614+091$ is one of the low-luminosity bursters. It was previously identified as an X-ray burster (Swank et al. 1978; Brandt et al. 1992; Brandt 1994) and as a probable atoll source (Singh \& Apparao 1995). BATSE monitoring has revealed numerous episodes of transient emission above $20 \mathrm{keV}$ from 4U 0614+091 (Ford et al. 1996a).

Here we describe the simultaneous observations of 4U 0614+091 using the Burst and Transient Source Experiment (BATSE) on board the Compton Gamma Ray Observatory (CGRO) and the all-sky monitor (ASM) on board the Rossi X-Ray Timing Explorer (RXTE). In 1996 April, with near-real-time BATSE monitoring, we discovered an episode of hard X-ray activity from 4U 0614+091, which was between 4 and 15 days long. This event provided a trigger for $R X T E$ pointings on Truncated Julian Day (TJD) 10,195 and 10,197, the results of which will be presented elsewhere (Ford et al. 1996b). The correlated ASM and BATSE observations presented here cover this 1996 April event. In $\S 2$, we describe the observations by both instruments. In $\$ 3$, we discuss the implications of these new results.

\section{OBSERVATIONS}

The analysis with BATSE uses the technique of Earth occultation, described, for example, in Harmon et al. (1992) 


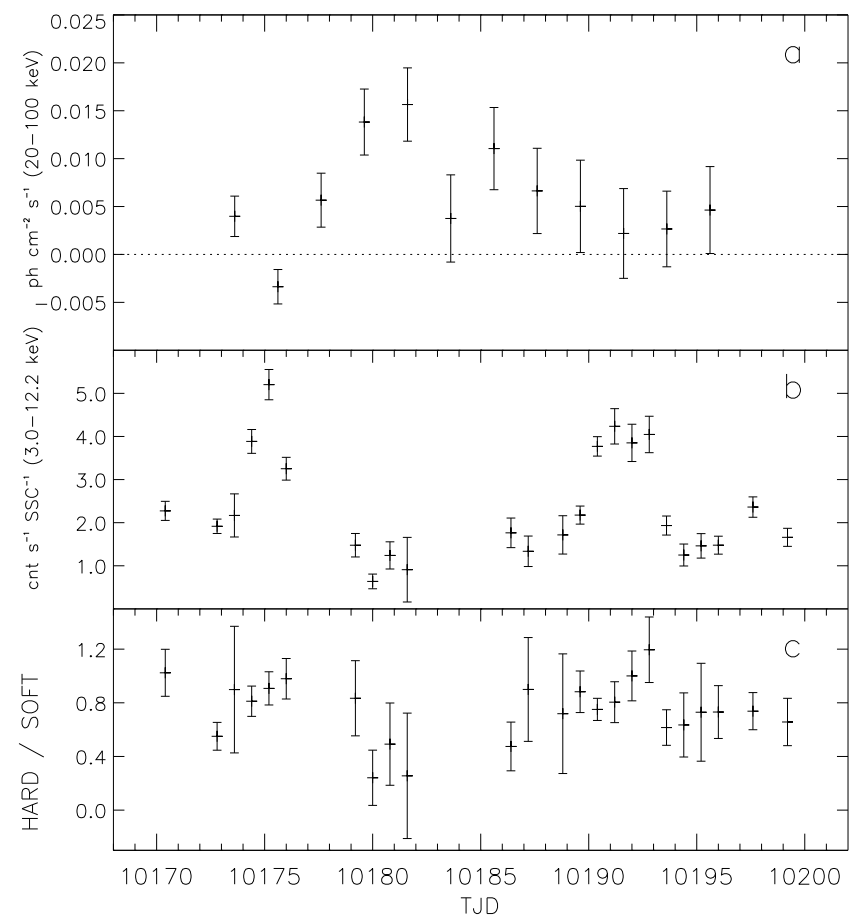

FIG. 1.-(a) BATSE and (b) RXTE ASM light curves. BATSE and ASM data are binned in 2 and 0.8 day bins, respectively. BATSE rate-toflux conversion assumes a power-law spectrum with index 2.8. (c) The [4.8-12.0 keV]/[1.3-3.0 keV] hardness ratio from the ASM data. TJD 10,175 corresponds to 1996 April 2.

and Zhang et al. (1993). This method offers a $20-150 \mathrm{keV} 3 \sigma$ sensitivity for 2 week integrations of approximately $1.7 \times 10^{-2}$ photons $\mathrm{cm}^{-2} \mathrm{~s}^{-1}(50 \mathrm{mcrab})$. With favorable detector geometries (when a source is near a detector normal) the sensitivity can improve to approximately $1.5 \times 10^{-2}$ photons $\mathrm{cm}^{-2} \mathrm{~s}^{-1}$ in only 2 days.

The ASM has been fully operational since early 1996 February ( TJD 10,132), following the launch of RXTE in 1995 December. The ASM consists of three Scanning Shadow Cameras (SSCs), which scan the sky about 7 times each day. The ASM detectors are position-sensitive proportional counters placed below coded masks. X-ray intensity measurements are derived from the deconvolution of overlapping mask shadows from each X-ray source in the field of view. The position histograms for each camera are separately recorded in three energy bands $(1.3-3.0 \mathrm{keV}, 3.0-4.8 \mathrm{keV}$, and $4.8-12.2$ $\mathrm{keV})$. The data are processed through standard filters, such as a $75^{\circ}$ cut on the Earth angle. In addition, for this analysis we consider only on-source dwells in which 4U 0614+091 is the only source in the field of view. The fine details of the instrument calibration are still being refined, and the present analysis is subject to a systematic error of about $3 \%$ for uncrowded source regions, as inferred from the rms deviations observed in the Crab Nebula. Further details of the instrument and the data analysis methods are provided by Levine et al. (1996).

The 4U 0614+091 light curves are shown in Figure 1. Integrating the BATSE fluxes (Fig. 1a) over TJD 10,17810,196 , the source is detected at a significance of $5.7 \sigma$. To confirm that the BATSE light curve is not contaminated by interfering sources, we have produced maps of the region around 4U 0614+091 during the time of the activity. Maps are

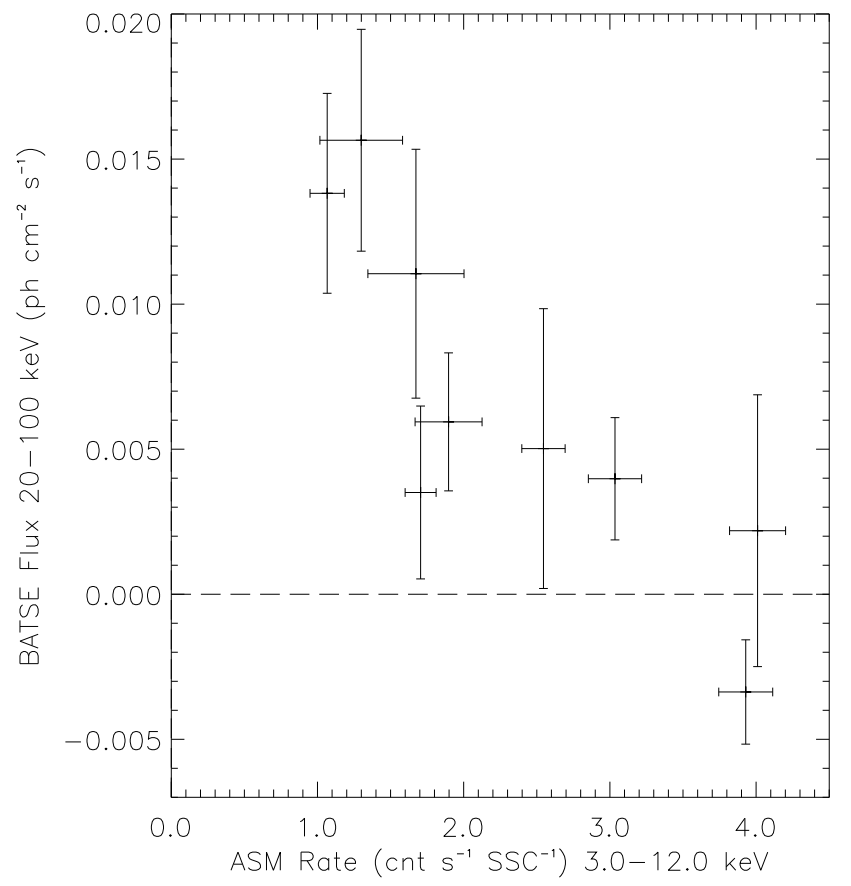

FIG. 2.-BATSE 20-100 keV flux vs. ASM 3.0-12.0 keV count rate. Data are binned in matching 2 day intervals.

generated with the occultation technique by producing rate histories over a grid of points (see Ford et al. 1996a). The maps show an obvious enhancement centered on the position of 4U 0614+091-clearly separated from the Crab, which lies approximately $17^{\circ}$ away. At periodic intervals, the limbs passing through $4 \mathrm{U} 0614+091$ also sweep over the Crab. This occurs for about 25 days prior to TJD 10,174 and after TJD 10,197. At these times, there is no information on the hard $\mathrm{X}$-ray flux.

Figure $1 b$ shows the ASM light curve (3.0-12.2 keV) binned into 0.8 day averages, contemporaneous with the BATSE activity. Two separate outburst peaks are clearly visible at TJD 10,175 and 10,191 . The gap in the ASM coverage near TJD 10,185 was caused by scheduling problems in the $R X T E$ operations.

A striking feature of Figure 1 is the anticorrelation of the BATSE flux with the ASM count rate. This anticorrelation is exhibited more clearly in Figure 2, which uses a common time binning of 2.0 days for the hard and soft fluxes. The anticorrelation is robust to changes in the energy bands.

We can define hardness ratios for the ASM fluxes, using the rates in the $1.3-3.0,3.0-4.8$, and $4.8-12.0 \mathrm{keV}$ bands $(R 1, R 2$, and $R 3$, respectively). The $R 3 / R 1$ hardness ratio is shown in Figure $1 c . R 3 / R 1$, and also the other hardness ratios, do not show a significant correlation with the source intensity. Colorcolor diagrams of the ASM data indicate that we cannot rule out a small contribution from a $1.0-1.5 \mathrm{keV}$ blackbody, as in Barret \& Grindlay (1995).

We have produced spectra from the ASM and BATSE data in the window TJD 10,178-10,190. During this entire interval, the source is in a "hard" state; the ASM count rate is low, while the BATSE flux reaches a peak. To generate the ASM spectrum, count rates were converted to fluxes by normalizing to the Crab rates observed by the ASM over the same period. The flux errors are calculated from the observed (Gaussian) 


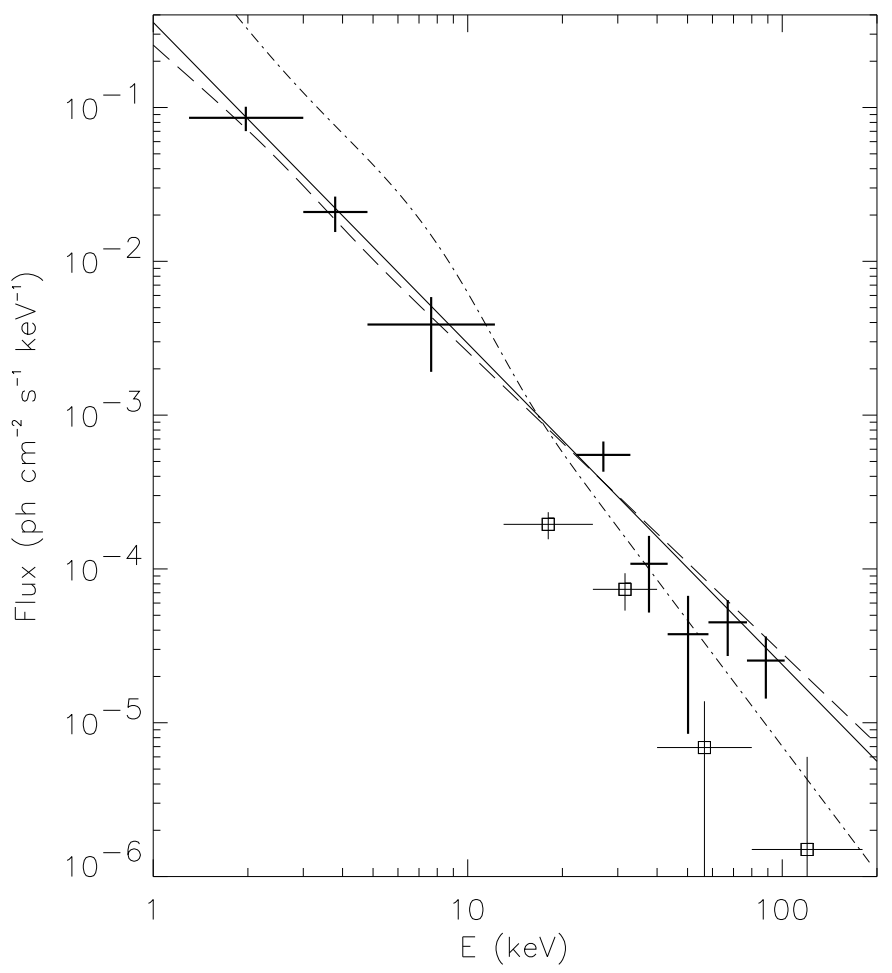

FIG. 3.-Combined BATSE and ASM spectrum over the period TJD $10,178-10,190$. Solid line is the single power-law fit $(\alpha=2.09 \pm 0.08)$ to the ASM and BATSE fluxes. Dashed (dot-dashed) lines show the low (high) states as identified by EXOSAT. These are extrapolated above the EXOSAT limit of $15 \mathrm{keV}$. Open squares are measurements by $H E A O 1 \mathrm{~A}-4$ from Levine et al. (1984).

distribution of the Crab count rate. For a conservative error estimate, we use the $3 \sigma$ value of the Crab rate distribution. The resulting flux errors for $4 \mathrm{U} 0614+091$ are approximately $10 \%$ in the log-log space used for spectral fitting. A power-law fit to the three ASM bins yields a spectral index of $2.23 \pm 0.33\left(\chi_{v}^{2}=0.05\right)$. The low $\chi^{2}$ results from the conservative flux error estimate. The averaged ASM spectrum corresponds to a 1-20 keV flux of $1 \times 10^{-9} \mathrm{ergs} \mathrm{cm}^{-2} \mathrm{~s}^{-1}$ with an implied luminosity of $1 \times 10^{36} \mathrm{ergs} \mathrm{s}^{-1}$ at $3 \mathrm{kpc}$.

The BATSE data in the same time window can be fitted by a power law with photon index $2.68 \pm 0.35\left(\chi_{v}^{2}=1.1\right)$. This fit is consistent with the spectra from an archival BATSE analysis of 4U 0614+091 during hard X-ray emission episodes (Ford et al. 1996a). The BATSE flux is $7 \times 10^{-10} \mathrm{ergs} \mathrm{cm}^{-2}$ $\mathrm{s}^{-1}(20-100 \mathrm{keV})$, corresponding to a luminosity of $8 \times 10^{35}$ ergs $\mathrm{s}^{-1}$ at $3 \mathrm{kpc}$.

Figure 3 shows the combined BATSE and ASM spectra in the TJD 10,178-10,190 window. A single power law with index $2.09 \pm 0.08$ can describe the combined data $\left(\chi_{v}^{2}=1.1\right)$. The index is harder than either of the two independent fits. We note that this spectral index is consistent with the range 1.86-2.24 for the low state, as observed with EXOSAT (Barret \& Grindlay 1995). Although it is difficult to test more complicated models, we attempt to fit a power law with a cutoff, which is an approximation to the Comptonization model in the optically thin regime. Fitting results are summarized in Table 1 .

To test the validity of combining ASM and BATSE spectra, we have generated spectra for the Crab over the same time interval. The spectra match to within $\sim 20 \%$, with the BATSE
TABLE 1

SPECTRAL FITS

\begin{tabular}{|c|c|c|c|}
\hline Model: Data & $\alpha$ & $\begin{array}{c}A_{0} \\
{\left[\times 10^{-3}\right]}\end{array}$ & $\chi_{\nu}^{2}, \nu$ \\
\hline PL: BATSE . & $2.68(0.35)$ & $7.05(3.57)$ & $1.1,3$ \\
\hline PL: ASM ........... & $2.23(0.33)$ & $2.30(1.06)$ & $0.05,1$ \\
\hline PL: BATSE+ASM $\ldots \ldots \ldots \ldots \ldots \ldots$ & $2.09(0.08)$ & $2.92(0.32)$ & $1.1,6$ \\
\hline CUTOFFPL: BATSE+ASM ......... & $1.79(0.18)$ & $3.83(0.97)$ & $1.8,5$ \\
\hline
\end{tabular}

NoTES.-Fits to data in the interval TJD 10,178-10,190. The spectral fits are of the form, PL: $A_{0}(E / 10 \mathrm{keV})^{-\alpha}$, CUTOFFPL: $A_{0}(E / 10 \mathrm{keV})^{-\alpha} e^{-E / E_{c}}$ (photons $\left.\mathrm{cm}^{-2} \mathrm{~s}^{-1} \mathrm{keV}^{-1}\right) . E_{c}=45(20) \mathrm{keV}$. Errors (parenthesis) are $1 \sigma$.

flux being higher than the ASM flux. The difference has a low $(\sim 1 \sigma)$ significance. With our conservative error estimates, the ASM fluxes are in agreement with the extrapolated BATSE fit with a $\chi^{2}$ of 0.93 .

\section{DISCUSSION}

Taken independently, our results in the soft and hard X-ray bands are consistent with previous observations. From 1 to $20 \mathrm{keV}, 4 \mathrm{U} 0614+091$ has been observed before at similar luminosities; e.g., $5 \times 10^{36} \mathrm{ergs} \mathrm{s}^{-1}$ at $3 \mathrm{kpc}$ with EXOSAT (Barret \& Grindlay 1995). In the hard X-ray band, numerous episodes of emission, similar to this one, have been identified in BATSE monitoring (Ford et al. 1996a). Also similar to what we find here, variability has been recorded by factors of approximately 5 on timescales of days. The $20-100 \mathrm{keV}$ flux, extrapolated from the EXOSAT spectrum in a high state, falls a factor of 4 below the peak of the present hard X-ray outburst and would not be detectable by BATSE. The EXOSAT low state closely matches the BATSE and ASM detections during the hard X-ray episode (Fig. 3).

This observation of $4 \mathrm{U} 0614+091$ is unique for its simultaneous broadband coverage. The most notable feature is the clear anticorrelation soft and hard X-ray emission (Fig. 2), which confirms a qualitative trend of anticorrelation noted earlier at lower energies in 4U 0614+091 and other bursters. The observed soft and hard anticorrelation indicates that either the soft or the hard X-ray emission is not a direct measure of the mass accretion rate. Analyzing short segments of ASM and BASTE data, we have found that the total energy flux in the $1-100 \mathrm{keV}$ band tracks the $1-12 \mathrm{keV}$ flux, while the hard 20-100 keV energy flux remains below $1 \times 10^{-9}$ ergs $\mathrm{cm}^{-2} \mathrm{~s}^{-1}$ with no observable correlation. This indicates that it is, in fact, the soft X-ray emission that tracks the mass accretion, as is usually assumed. The hard X-ray flux, therefore, is actually anticorrelated with mass accretion rate, and is most likely a manifestation of a special property of the accretion disk. We note that in black hole candidates-for example, Cyg X-1 (Crary et al. 1996)—the hard X-ray emission is also believed to be anticorrelated with the mass transfer rates.

Both thermal (Comptonization) and nonthermal models of emission can be considered in explaining the hard X-ray emission properties of $4 \mathrm{U} 0614+091$. A thermal model (e.g., Sunyaev \& Titarchuk 1980) could account for the hard and soft anticorrelation in the following way: when the soft X-ray flux increases, the Comptonizing plasma cools, resulting in a decreased temperature and hard X-ray flux. Nonthermal models have also predicted hard X-ray emission from neutron stars (Kluzniak et al. 1988) and may explain our results. In nonthermal models, the soft and hard X-ray anticorrelation is the 
result of the suppression of particle acceleration by inverse Compton cooling caused by an enhanced soft X-ray background. Only for intermediate to low values of $L_{s}$ can the hard $\mathrm{X}$-ray emission manifest itself as a consequence of a nonthermal particle energy distribution function of energized particles in the disk (Tavani \& Liang 1996).

These 4U 0614+091 results are a complement to recent observations of the X-ray burster 4U 1608-522 (Zhang et al. 1996). 4U 1608-522 showed a bright ( 100 mcrab) outburst in the BATSE light curve that lasted approximately 200 days. During this outburst, the source was detected by Ginga in a low state, making it the first NS binary in which a low soft $\mathrm{X}$-ray state was definitively linked to a bright phase in hard X-rays. Our observations of $4 \mathrm{U} 0614+091$ extend these results, showing that the hard and soft fluxes are anticorrelated as the source varies. The spectrum of $4 \mathrm{U} 1608-522$ exhibits a clear break at approximately $65 \mathrm{keV}$ with spectral indices 1.8 and $3.2 \pm 0.2$. For $4 \mathrm{U} 0614+091$, the spectra may roll over at a lower energy, which would have important implications for the hard X-ray emission models. This could point, for example, to lower electron temperatures in thermal models.

The present data indicate that when the $R X T E$ pointed observations were performed on TJD 10,195 and 10,197, the source was in a low state with a hard power-law spectrum $(\alpha \sim 2.1)$ and little contribution from a soft component.
During these observations, we have discovered two highfrequency QPOs over the range $580-950 \mathrm{~Hz}$ (Ford et al. 1996c). The QPO frequency and total count rate are linearly correlated, while the frequency difference between the two peaks is constant. Perhaps the presence of $\mathrm{kHz}$ QPOs is linked to the hard X-ray brightness. The two other atoll sources with kHz QPOs, 4U 1728-34 and 4U 1608-52 (Strohmayer et al. 1996; van Paradijs et al. 1996), have also been detected as bright hard X-ray sources by BATSE (Barret et al. 1996).

\section{CONCLUSION}

Combining the capabilities of BATSE Earth occultation monitoring and the ASM on board RXTE, we have measured the flux of $4 \mathrm{U} 0614+091$ over a wide energy band (1-100 keV) during several months. We detected an active hard X-ray phase accompanied by soft X-ray variability. The hard and soft $\mathrm{X}$-ray fluxes show a distinct anticorrelation. This measurement is an example of the new opportunities now available for broadband X-ray monitoring.

We would like to acknowledge the BATSE instrument team and the members of the $R X T E$ Guest Observer Facility for their support and assistance. This work is supported in part by NASA grants NAG 5-2235 and NGT 8-52806.
Barret, D., et al. 1996, in Third Compton Symp., A\&AS, in press

Barret, D., \& Grindlay, J. 1995, ApJ, 440, 841

Barret, D., \& Vedrenne, G. 1994, ApJS, 92, 505

Bouchacourt, P., et al. 1984, ApJ, 285, L67

Brandt, S. 1994, Ph.D. thesis, Danish Space Research Institute

Brandt, S., et al. 1992, A\&A, 262, L15

Breedon, L. M., et al. 1986, MNRAS, 218, 487

Crary, D. J., et al. 1996, ApJ, 462, L71

Ford, E., et al. 1996a, in preparation $1996 b$, in preparation
1996 , IAU Circ. 6426

Harmon, B. A., et al. 1992, in Compton Observatory Science Workshop, NASA CP, 3137, 69

Inoue, H. 1990, Adv. Space Sci., 10(2), 153

Kluzniak, W., Ruderman, M., Shaham, J., \& Tavani, M. 1988, Nature, 336, 558

Langmeier, A., et al. 1987, ApJ, 323, 288

\section{REFERENCES}

Levine, A. M, Bradt, H., Cui, W., Jernigan, J. G., Morgen, E. H., Remillard, R. A., Shirey, R., \& Smith, D. 1996, ApJ, 469, L33

Levine, A. M., et al. 1984, ApJ, 54, 581

Mitsuda, K., et al. 1989, PASJ, 41, 111

Singh, K. P., \& Apparao, K. M. V. 1995, ApJ, 431, 826

Smale, A. P., Corbet, R. H. D., Charles, P. A., Menzies, J. W., \& Mack, P. 1986, MNRAS, 223, 207

Strohmayer, T., et al. 1996, in IAU Circ.6320

Sunyaev, R. A., \& Titarchuk, L. 1980, A\&A, 86, 121

Swank, J. H., et al. 1978, MNRAS, 182, 349

Tanaka, Y. 1989, in Proc. 23rd ESLAB Symp. 1, ed. J. Hunt (Paris: ESA), 3

Tavani, M., \& Liang, E. 1996, in 3rd Compton Symp., A\&AS, in press

van der Klis, M. 1994, ApJS, 92, 511

van Paradijs, J., et al. 1996, IAU Circ. 6336

Zhang, S. N., et al. 1993, Nature, 366, 245

Zhang, S. N., et al. 1996, in 3rd Compton Symp., A\&AS, in press 